\title{
A general and flexible model for the pedagogical description of learning objects
}

\author{
Serena Alvino ${ }^{1}$, Paola Forcheri ${ }^{2}$, Maria Grazia Ierardi ${ }^{2}$ and Luigi Sarti ${ }^{1}$ \\ ${ }^{1} \mathrm{CNR}$, Istituto per le Tecnologie Didattiche \\ Via De Marini 6, 16149 Genova, Italy \\ \{alvino,sarti\}@itd.cnr.it \\ WWW home page: http://www.itd.cnr.it \\ ${ }^{2}$ CNR, Istituto Di Matematica Applicata e Tecnologie Informatiche \\ Via De Marini 6, Genova, Italy \\ \{forcheri,marygz\}@ge.imati.cnr.it \\ WWW home page: http://www.ge.imati.cnr.it
}

\begin{abstract}
We illustrate a pedagogical metadata model (IMATI-ITD pedagogical metadata model) that captures didactic features of LOs according to the view of the education world. This model allows to describe a variety of contexts and can be effectively instantiated in many specific educational situations, thus combining generality with flexibility features.
\end{abstract}

\section{Introduction}

Learning object (LO) technology still struggles to gain momentum and acceptance in the communities of teachers and instructional designers; in fact, the considerable effort needed for realising easily reusable material and for integrating others' products in a specific pedagogical plan makes the sharing of didactical material a difficult task [1]. To support users' motivation to invest their time and efforts in this activity, notable efforts are devoted to turning repositories of LOs (LORs) into a basis for building learning communities of teachers and trainers [2]. A recent example is the SLOOP project (http://www.sloopproject.eu/): it fosters the development of such communities by integrating in a LOR functionalities aimed at supporting the enrichment of preexisting material. Another proposal is LODE, an open source collaborative environment built around a LOR and based on an interactive model of the reuse activity [3].

The work carried out produced very valuable results. However, its effective application in the education realm requires LO producers to provide users with a clear pedagogical picture of the material [4]. As a matter of fact, it is generally recognised that present metadata standards, like IEEE LOM [4], have a limited capacity to offer 
this picture according to the view of the education world [6]. To overcome this problem, several studies analyse the problem from the perspective of the education practice: some suggest methods for defining pedagogical metadata based on the analysis of the prospective users (e.g., [7]); others propose application profiles including descriptors that are selected according to the orientation and objectives of specific communities of users (e.g.: GEM http://hegateway.org/, EDNA http://www.edna.edu.au/). These studies show the complexity of devising a pedagogical metadata model apt at being successfully employed by a variety of user communities, as these are usually characterised by different languages, backgrounds, motivations and objectives. The solution of this problem requires, in our view, a model that combines generality with flexibility, so to be sharable among different communities and, at the same time, adaptable to the specificity of each of them.

Our work gives a contribution in this direction. Starting from an initial proposal, we adopted an iterative approach to the evaluation of its adherence to the needs of a number of communities. We then elaborated an extensible model for pedagogical metadata (IMATI-ITD pedagogical metadata model), that includes: 1) a set of descriptors, generally sharable among communities, that constitutes the common framework for capturing LO features; 2) for each community and each descriptor, a vocabulary of possible values that represent (as names and meanings) the conception of the descriptor within that specific community.

Metadata provide the underpinnings of our model. For each metadata element the set of possible values represents the union of the conceptions of the various communities. To make the proposal effectively applicable, moreover, the model is integrated with a set of tools that allow users, in both the indexing and the retrieval phases, to obtain a personalized view of the model, representing its adaptation to the community at hand. Adaptable glossaries are also provided.

The model and the approach we followed in devising it are presented in this paper. We observe, preliminarily, that the model, focusing on the pedagogical aspects, is not intended to describe content-related aspects, for which we refer to international standard schemata.

\section{Describing LOs' pedagogical dimension}

\subsection{Conceptual approach}

The conceptual design of our pedagogical model (see Fig. 1) is based on the observation that teachers, in their work, make use not only of material to be employed directly with students, but also of schemata, scripts, meta-models that support them in developing educational proposals. Material of this type, oriented to plan activities such as for example a web-based discussion between peers, or a collaborative project, is a very valuable reusable educational resource, as it constitutes a sort of guide to carry out innovative classroom activities.

Pedagogical metadata, as a consequence, should help the teacher to efficiently select LOs that are representative of two kinds of materials: resources, oriented to students, 
aimed at being used directly in the education practice; and resources, oriented to teachers, aimed at organising it (see Fig. 1).

Depending on the type of help offered to teachers, on the level of abstraction from the context and on the level of formalization, teacher-oriented resources are of various nature: 1) Units of Learning (UoL), representing such resources as lessons, modules or entire courses described with formal languages (EML - Educational Modelling Languages; see for instance the IMS Learning Design specification: http://www.imsglobal.org/learningdesign/index.cfm); 2) Pedagogical patterns and the like, representing resources that model specific strategies or techniques to solve classes of educational problems, thus making good practices transferable [8]; 3) Lesson plans, representing resources that illustrate teaching proposals, instantiated in a specific knowledge domain, in terms of objectives, strategies, supporting tools, resources, possible uses and so on; they are formulated in plain natural language and no constraints are imposed on their structure.

As we already discussed in [9], learner-oriented resources, in turn, can be classified depending on the role they are intended to play in the learning process. In particular, we consider educational module, endowed with well-identified educational objectives and pedagogical approaches, so we call them Structured $L O$. We also include auxiliary materials (report forms, FAQs, bibliographies, etc.), which have a generalpurpose or context-related function: we call them Functional LOs.

From the point of view of the pedagogical structure, Structured LOs and Teacher oriented LOs are endowed with (or suggest, in case of Teacher oriented LOs) an articulated pedagogical architecture; moreover, they are inspired by precise assumptions on learning and implement (or offer a model for realising) a specific learning strategy. Functional LOs, on the contrary, feature limited pedagogical orientation (for example, a bibliography comprises references suitable to the learner level and background, but it does not include an explicit pedagogical strategy). The pedagogical description of Functional LOs, thus, partly differs from that of the other kinds of LOs.

\subsection{Realisation}

The IMATI-ITD pedagogical metadata model realises the above illustrated view by integrating descriptors from the main international metadata standards with new ones aimed at identifying the context of use, the educational features, the structure and the learning approach of the resource. More precisely, our proposal is organized in five categories of metadata: Pedagogical Model, General, Audience, Educational Features, Annotation (see Fig. 1).

The category Pedagogical Model constitutes the characteristic feature of our proposal. It includes a descriptor, Type, that differentiates LOs according to the ideas discussed in Section 2.1. This element is mandatory and for any given LO it can assume one and only one of the values of its vocabulary. Moreover, the descriptor Type acts as a selector of the other descriptors in the Pedagogical Model category:

- Functional LOs are described on the basis of the typology of the resource (ResourceType); the values for this descriptor include, for instance, FAQ list, game, movie, computer simulation etc. 
- Structured LOS are described on the basis of the pedagogical orientation, the didactic strategy, the type of activity proposed and the (self)-assessment material, if any. The element aimed at describing the pedagogical orientation (SubType) relies on a single-choice set of values: Guided, if the educational resource aims to introduce the student into a new topic under a planned guidance; Problem, if it proposes a problem situation to be explored autonomously by the student; Mixed, if it integrates the two approaches [9]. A vocabulary of thirteen multiple-choice values is provided for the element describing the didactic strategy.

- Teacher-oriented LOs are described similarly to Structured LOs. In this case, however, descriptors refer to activities, lessons, didactic units or modules that the LO proposes.

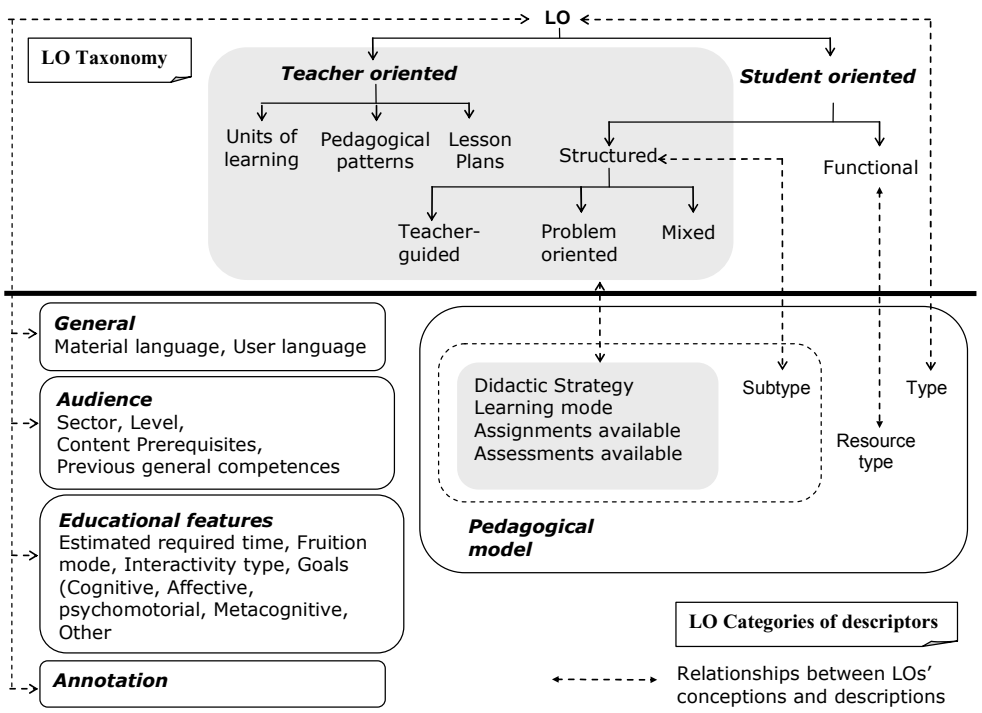

Fig. 1. LO conceptions and their corresponding descriptions.

The category General includes two descriptors analogous to the LOM descriptors 1.3 General.Language and 5.11 Educational.Language respectively [4].

The category Audience describes the characteristics of the intended user of a LO (in case of teacher-oriented LOs this is the final user, i.e. the learner). It includes elements, similar in their meanings to analogous descriptors of other proposals, to describe the sector and level of the intended student. Differently from other proposals, this category also comprises elements to describe prerequisites that refer not only to the content domain but also to the general competences that should be mastered to successfully deal with the LO.

The category Educational Features describes features such as the time estimated necessary for the fruition of the LO (by means of a descriptor with the same meaning and values as the LOM 5.9 Typical learning time element) and the fruition mode (presence, distance or blended). Other elements in this category describe the nature of the interaction that is to be activated by the LO and the physical environment (for 
example lab, museum, classroom ...) that is more suitable to foster its effective fruition. We also include in this category the educational goals. Differently from other proposals, we consider not only the cognitive domain of Bloom's taxonomy [10], but also the psychomotor and affective ones. We also add descriptors for meta-cognitive goals and for specific school goals (for example those connected with the relationship with the parents of 6 to 11 years old students in the context of Italian primary schools).

The category Annotation corresponds to the LOM Annotation category.

For the technical details of the IMATI-ITD model we refer to [11].

\section{The validation process}

We intended to develop a pedagogical metadata model that would meet as much as possible the needs of a variety of teachers communities. To this aim, we designed a preliminary version based on both our previous experience and on widespread LO metadata specifications, such as the Learning Object Metadata (LOM) schema [4], the Gateway to Educational Materials (GEM, http://thegateway.org) project proposal and the one formulated by the Education Network Australia (EdNA, http://www.edna.edu.au/).

We then carried out a validation process of the effectiveness of our choices with four groups of prospective users, each group belonging to a different educational realm. The user groups we considered were: 1) practitioners in the academic field, represented by instructional designers, e-learning experts and faculty members (14 persons); 2) pre-service teachers and trainers, represented by M. S. students, attending a basic course in "E-learning and knowledge management" (20 persons), and student teachers attending the postgraduate Specialisation School for Teaching in Secondary Schools (80 persons); 3) vocational instructors, represented by professionals working in a large European insurance company (8 persons); 4) primary school teachers, represented by schoolmistresses involved in a project aimed at developing a repository of animation-based LO for 6 to 11 years old children (34 persons).

The validation process was carried out iteratively. Each step provided us with feedbacks and suggestions from the specific group of users involved; on this basis, we refined and enriched the model. We gathered heterogeneous hints, since we integrated two different validation strategies:

- An experimental approach. By using a web-based tool that was designed and implemented to this purpose, participants had to index some LOs and to write down notes about the critical aspects that emerged. Validation focused on the elements, specific of our proposal, whose values are listed in a closed vocabulary. After indexing the LOs, each participant was individually interviewed. The results of the interviews were used as a basis to progressively modify the model.

- A participatory approach [12] was used in the case of primary school teachers. It allowed us to set a validation context fostering the negotiation of meanings and the sharing of different points of view. The process was developed as a distant cooperation supported by frequent audio-conferencing meetings, interspersed with local group meetings. This work started with an initial step of "grounding", aimed at setting the workspace, sharing specific objectives and defining a shared language to 
talk about the problem. Teachers participated actively into decision making and helpfully contributed to adapt the model to the requirements of their activity and situation, still taking into account the general purpose of the model.

The validation strategy was very effective in negotiating a shared view on the elements of the model, which the teachers accepted and actively contributed to improve. We refer to [13] for a discussion of the results. For the sake of space, here we limit ourselves to summarise (see Table 1) the appreciation for the model, resulting from the overall validation process, shown by 80 prospective teachers.

Table 1. Appreciation of the general features of the model ( $\%$ of respondents). Respondents: 80 prospective teachers.

\begin{tabular}{|c|c|c|c|c|}
\hline & & YES & NO & no answer \\
\hline Model & Does the model capture the LO educational features? & 95,00 & 3,75 & 1,25 \\
\hline \multirow{4}{*}{$\begin{array}{l}\text { IMATI-ITD } \\
\text { Metadata }\end{array}$} & Are metadata names clearly understandable? & 77,50 & 22,50 & 0,00 \\
\hline & Would you add other metadata? & 13,75 & 85,00 & 1,25 \\
\hline & Would you remove some metadata? & 15,00 & 83,75 & 1,25 \\
\hline & Are metadata useful for searching for LOs? & 88,75 & 10,00 & 1,25 \\
\hline \multirow{2}{*}{$\begin{array}{l}\text { The metadata } \\
\text { Pedagogical } \\
\text { Model.Type }\end{array}$} & $\begin{array}{l}\text { Does its values represent, from a methodological point } \\
\text { of view, all possible types of LOs? }\end{array}$ & 95.00 & 5,00 & 0,00 \\
\hline & $\begin{array}{l}\text { Is it effective the mandatory choice of one and only } \\
\text { one value for describing a LO? }\end{array}$ & 80,50 & 19,50 & 0,00 \\
\hline \multirow[t]{3}{*}{ Vocabularies } & Are vocabularies clear? & 78,75 & 20,00 & 1,25 \\
\hline & Would you add values in any vocabulary? & 11,25 & 87,50 & 1,25 \\
\hline & Would you eliminate values in any vocabulary? & 10,00 & 88,75 & 1,25 \\
\hline
\end{tabular}

\section{Discussing the model and the validation process}

Some results of the validation process summarized in Table 1 seem to indicate that our proposal is quite a valuable attempt to describe the pedagogical aspects of LOs. However, some critical elements emerged that regard the usability of the model. Sometimes users find it difficult to univocally interpret specific terms of the model. For instance, when dealing with the element Type of the category Pedagogical Model, users belonging to different communities interpret differently the meaning of the structured vs. functional distinction. Regardless of the web-based tool's functionalities aimed at supporting the user's choices (e.g., a glossary or an on-line help), ambiguities arise because of differences in specific community sub-cultures and jargons. For instance the same LO can be regarded as a simulation by some, or as a case study by others. Of course, there is also a linguistic problem related to the translation of most of the terms used in the model into the user's native language.

Another critical aspect is the level of detail captured by some vocabularies: if, on one side, it reflects the expressiveness of the model, on the other side, it often needs to be tuned to specific user needs. This is particularly evident with the Audience.level element: primary school teachers, for instance, need to distinguish between two-year periods in the curriculum, whereas practitioners in adult and lifelong learning are not concerned with such fine-grain distinctions, which are regarded as noise. Similarly, the 
Audience.sector element calls for quite a refined vocabulary in the university context, and for a more limited one in primary school. Furthermore, both these elements heavily depend on social, cultural and national systems: even at the same university level curricula are likely to differ depending on the nations, thus requiring adaptations of the Audience.sector vocabulary.

The above considerations highlight the need of adapting both element names and vocabularies to the requirements of specific communities of users. Accordingly, a twolayer architecture has been identified.

The abstract metadata model is defined as a namespace schema [14] that acts as a foundation for a number of application profile schemata, each one tailored and optimized for a specific user community. The application profiles can use elements from the complete model, refine the definitions and narrow down vocabularies, but they cannot create new elements nor extend the original vocabularies.

In our approach an application profile is defined on the basis of the full metadata model through the following steps: 1) the subset of descriptor elements that suits the expressive needs of the target community is identified (care must be paid to maintain the structural consistency of the resulting (sub)schema); 2) if necessary, the names of the identified elements are translated into the community's native language; 3 ) for each vocabulary, a subset of items is identified similarly to point 1 above and, if required, translated into the target language. This process is not free from hindrances and pitfalls, and requires the involvement of representatives of the user communities in a participative effort.

\section{Future perspectives}

According to the Dublin Core Metadata Initiative (DCMI) Glossary (http://dublincore.org/documents/usageguide/glossary.shtml), "an application profile is not complete without documentation that defines the policies and best practices appropriate to the application". Such a documentation can only be built upon an extensive set of experimental, situated activities aimed at validating the model in as many and various contexts as possible, with the direct involvement of users: this will be the direction of our future research task.

The experiments described above relied on hand-coded user interfaces; extending the scope of the validation will call for a better articulation of the supporting tools. A formal representation of the model is the next step: by developing an XML schema for the abstract model it will be possible to automate, to some extent, the task of generating the repository structure, the user interface software and the representation of the new application profile. Suitable software modules will support the selection of elements and vocabulary items that constitute the application profile, and they will automatically produce its formal representation, the logical schema of the database hosting the LO repository, and the skeleton of the interface applications that support users in loading and querying the repository. One possible evolution path would involve the extension of the architecture outlined in section 4 with a third layer, namely the interface one. As a matter of fact, some minor adaptation issues could be dealt with by the user interface, thus limiting the uncontrolled proliferation of slightly 
differing application schemata. On the other hand, encapsulating the model specialization into a formally defined application schema can improve reusability and interchange among different repositories.

"The limits of my language indicate the limits of my world" ([15], prop. 5.6). Language supports thinking, and even such a confined linguistic tool as a metadata description model can influence the way people look at educational material, enhancing their ability to capture and represent meaning and refining their selection skills.

\section{Acknowledgments}

This work was supported in part by the Italian Ministry of University and Research, Project VICE (Virtual Communities for Learning). The authors are grateful to all the people who contributed to the model validation process for their precious suggestions.

\section{References}

1. T. Hand, Learning Objects: User Perspectives on the Conditions Surrounding Their Use. In L. Cantoni \& C. McLoughlin (Eds.), Proc. of World Conference on Educational Multimedia, Hypermedia and Telecommunications 2004. Chesapeake, VA: AACE, 66-72.

2. O. Liber, Learning objects: conditions for viability, J. Comp. Assisted Learning, 21(5), 366373, 2005.

3. G. Dettori, P. Forcheri, and M.G. Ierardi, Endowing LOs with a social dimension. In ICWL 2006, Wenyin Liu, Qing Li, Rynson W. H. Lau, LNCS, Springer 2006, 189-202.

4. A. Littlejohn, Reusing online resources, Kogan Page, London. 2004.

5. IEEE Learning Technology Standards Committee, Learning Object Metadata, Final Draft Standard, IEEE 1484.12.1-2002. Available in: http://ltsc.ieee.org/wg12, 2002.

6. J. Griffith, G. Stubbs, and M. Watkins, From course notes to granules: A guide to deriving Learning Object components, in Computers in Human Behavior, 23(6), 2007, 2696-2720.

7. B. Simon, and J. Quemada, A Reflection of Metadata Standards Based on Reference Scenarios", in Proc. der GMW, Tagung 2002, 2002.

8. P. McAndrew, P. Goodyear, and J. Dalziel, Patterns, designs and activities: unifying descriptions of learning structures, I. J. of Learning Technology, 2(2-3), 2006, 216-242.

9. E. Busetti, G. Dettori, P. Forcheri, and M.G. Ierardi, A pedagogical approach to the design of learning objects for complex domains, I. J. Distant Educ. Technologies, 5(2), 2007, 1-17.

10. B.S. Bloom,Taxonomy of Educational Objectives. The Classification of Educational Goals, in Handbook I, Cognitive Domain. B. S. Bloom, Ed. New York: Longman, 1956.

11. S. Alvino, P. Forcheri, M.G. Ierardi, and L. Sarti, Un modello per la descrizione pedagogica di learning objects, Progetto MIUR-VICE, Deliverable 2007.

12. T. Winograd, Bringing design to software (Addison-Wesley, Boston, MA, 1996).

13. S. Alvino, P. Forcheri, M.G. Ierardi, and L. Sarti, Describing learning features of reusable resources: a proposal, International Journal of Social Sciences 2 (3), 2007, 156-162.

14.J. Mason, and H. Galatis, Theory and practice of application profile development in Australian education and training, Proc. Int'l Conf. on Dublin Core and Metadata Applications, 2007.

15. Wittgenstein, L., Logisch-Philosophische Abhandlung, Annalen der Naturphilosophie, 14, 1921. Trad. It.: Conte A.G. (a cura di) Tractatus logico-philosophicus e Quaderni 19141916, Einaudi, Torino, 1964. 